\title{
Vascular-wall remodeling of 3 human bypass vessels: Organ culture and smooth muscle cell properties
}

\author{
Armand Mekontso-Dessap, MD, a,c Matthias Kirsch, MD, PhD, ${ }^{\mathrm{b}}$ Christophe Guignambert, ${ }^{\text {a }}$ Patricia Zadigue, ${ }^{a}$
} Serge Adnot, MD, PhD, ${ }^{a}$ Daniel Loisance, MD, ${ }^{b}$ and Saadia Eddahibi, $\mathrm{PhD}^{\mathrm{a}}$

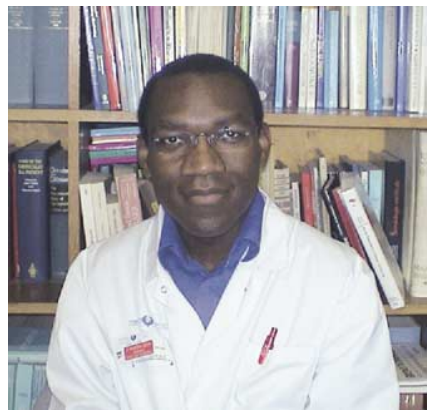

Dr Mekontso-Dessap

From Institut National de la Santé et de la Recherche Médicale, Faculté de Médecine de Créteil, ${ }^{\text {a }}$ Créteil, France, Service de Chirurgie Thoracique et Cardiovasculaire, Hôpital Henri Mondor, Assistance Publique-

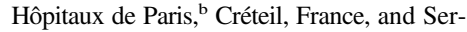
vice de Réanimation Médicale, Hôpital Henri Mondor, ${ }^{\mathrm{c}}$ Créteil, France.

Received for publication May 5, 2005; revisions received Aug 19, 2005; accepted for publication Aug 26, 2005.

Address for reprints: Armand MekontsoDessap, MD, Service de Réanimation Médicale, Hôpital Henri Mondor, Avenue du Maréchal de Lattre de Tassigny, 94000 Créteil, France (E-mail: armand.dessap@ creteil.inserm.fr)

J Thorac Cardiovasc Surg 2006;131:651-8 $0022-5223 / \$ 32.00$

Copyright () 2006 by The American Association for Thoracic Surgery

doi:10.1016/j.jtcvs.2005.08.048
Objectives: Late graft occlusions after coronary artery bypass grafting have been ascribed to neointimal hyperplasia. Given the pivotal role of smooth muscle cells in the pathogenesis of neointimal hyperplasia and the phenotypic heterogeneity of smooth muscle cells across vessels, we hypothesized that differences in long-term graft patency are at least partly related to differences in smooth muscle cell properties. The aim of the present study was to compare the vascular-wall remodeling of human internal thoracic artery, radial artery, and saphenous vein bypass conduits.

Methods: We evaluated the intimal thickening of the human graft segments in organ cultures (histopathology, morphometric, and immunofluorescence analyses) and assessed the properties of cultured smooth muscle cells isolated from these vessels in terms of cell proliferation (tritiated thymidine incorporation), migration (modified Boyden chamber), and collagen synthesis (tritiated proline incorporation).

Results: The total vessel-wall growth index and the intimal growth index were significantly higher for saphenous vein rings than for radial artery and internal thoracic artery rings. Immunofluorescence analyses showed predominant involvement of smooth muscle cells in neointimal growth induced by organ culture of saphenous vein rings. Cell proliferation was significantly higher in saphenous vein smooth muscle cells than in radial artery smooth muscle cells and significantly higher in radial artery smooth muscle cells than in internal thoracic artery smooth muscle cells. Migration of smooth muscle cells from saphenous vein grafts was significantly greater than from internal thoracic artery or radial artery grafts. Collagen synthesis was similar in smooth muscle cells from internal thoracic artery, radial artery, and saphenous vein grafts.

Conclusions: Ex vivo vascular-wall remodeling and smooth muscle cell intrinsic growth and migratory properties are dissimilar between arterial and venous grafts and might shed light on reported angiographic patency rates of these grafts.

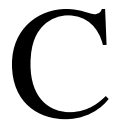
oronary heart disease is the most common cause of morbidity and mortality in industrialized countries. Coronary artery bypass grafting (CABG) with a combination of arterial and venous grafts is now a component of the standard treatment armamentarium for patients with coronary heart disease. CABG reduces both symptoms and mortality, particularly in patients with severe disease. However, its long-term benefits are dependent on continuing patency of the bypass conduits.

The long-term patency of saphenous vein (SV) grafts is limited, and approximately half of these conduits undergo occlusion within 10 years. ${ }^{1}$ These late occlusions have been ascribed to neointimal hyperplasia (NIH), with an accumulation of smooth muscle cells (SMCs) and extracellular matrix in the graft intima. NIH narrows the graft lumen and sets the scene for subsequent graft atherosclerosis. ${ }^{2}$ 


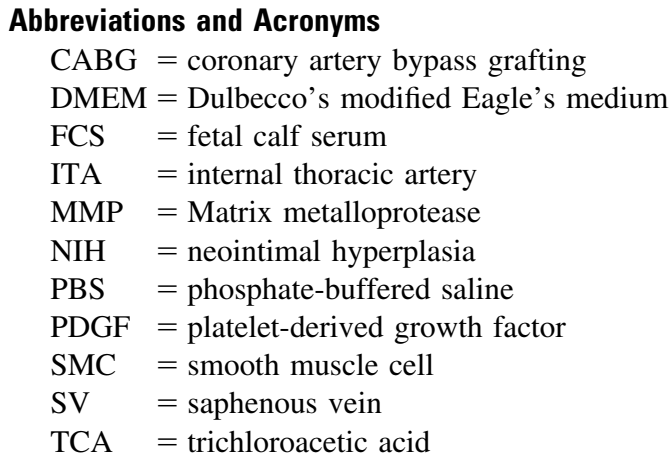

Compared with SV grafts, internal thoracic artery (ITA) grafts have yielded significantly better long-term results. ${ }^{3}$ This has prompted a search for other reliable arterial conduits. The radial artery (RA) was introduced by Carpentier and colleagues in 1973 as an alternative bypass conduit for CABG. However, it was soon abandoned because of its high failure rate attributed to spasm of the denervated artery and NIH formation. ${ }^{4,5}$ Nonetheless, improved harvesting techniques with avoidance of mechanical dilation, postoperative vasospasm prophylaxis, and appropriate grafting strategy have revived the radial artery as a conduit for coronary revascularization. ${ }^{6}$ In fact, several groups have recently reported better long-term clinical and angiographic results than with SV grafts. ${ }^{7-10}$

Given the pivotal role of SMCs in the pathogenesis of $\mathrm{NIH}$ and the well-established phenotypic heterogeneity of SMCs across vessels, ${ }^{11}$ we hypothesized that differences in long-term graft patency are at least partly related to differences in SMC growth and migratory properties. To investigate this hypothesis, we compared the remodeling of human ITA, RA, and SV bypass conduits using 2 approaches. First, we evaluated the intimal thickening of the ITA, RA, and SV segments in organ cultures. Second, we studied the properties of cultured SMCs isolated from ITAs, RAs, and SVs in terms of cell proliferation, migration, and collagen synthesis.

\section{Methods \\ Patients}

Discarded distal segments of ITAs, RAs, and SVs were obtained from consecutive patients undergoing CABG. Premedication, anesthesia, and intraoperative heparinization were done according to the usual protocol applied in our department of cardiac surgery. Patients gave their informed consent to ex vivo use of their blood vessel specimens. Material from 10 men and 2 women were sufficient for organ culture and cell culture. All 3 vessel types were not available for all patients. However, for each patient, at least 2 vessel types were available for each experiment. Mean age was $67 \pm 13$ years. Cardiovascular risk factors included diabetes $(n=4)$, dyslipidemia $(n=6)$, smoking $(n=6)$, a family history of coronary artery disease $(n=4)$, and hypertension $(\mathrm{n}=6)$.

\section{Organ Culture, Histopathology, and Morphometric Analysis}

After removal, the vessels were immediately immersed in Dulbecco's modified Eagle's medium (DMEM), placed at $4^{\circ} \mathrm{C}$, and transferred to the laboratory within 24 hours. Two adjacent rings 5 to $8 \mathrm{~mm}$ in length were dissected from each vessel. The first ring (R1) was immediately fixed without being cultured and served as the control. The second ring (R2) was fixed after 10 days of incubation in cell-culture flasks containing DMEM supplemented with $10 \%$ fetal calf serum (FCS), $2 \mathrm{mmol} / \mathrm{L}$ L-glutamine, and antibiotics $(100 \mathrm{U} / \mathrm{mL}$ penicillin and 0.1 $\mathrm{mg} / \mathrm{mL}$ streptomycin) at $37^{\circ} \mathrm{C}$ in a $5 \% \mathrm{CO}_{2}$ humidified atmosphere. The medium was changed every 2 days. For microscopic studies, vessel rings were fixed in $4 \%$ formaldehyde solution. Multiple transverse slides were processed in paraffin wax. Sections $5 \mu \mathrm{m}$ in thickness were cut and stained with hematoxylin and eosin, elastic stain, and orcein-picroindigocarmine. The slides were examined with a Charge-Coupled Device Iris camera (CDD Iris; Sony, Paris, France) coupled with an optical microscope (Laborlux, Wetzlar, Germany), and images were obtained with Perfect Image software (Clara Vision, Orsay, France). The total vessel wall area and the intimal area were measured on R1 and R2. The intima of ITAs and RAs was defined as the zone between the endothelium and the internal elastic lamina, and the intima of SVs was defined as the layer of tissue between the endothelium and the media. The vessel-wall growth index was used to compare preculture sections of each vessel with corresponding cultured sections and was computed as follows:

\section{( $R 2$ total vessel wall area $-R 1$ total vessel wall area)/}

$R 1$ total vessel wall area.

In addition, the intimal growth index was computed as follows:

$$
(R 2 \text { intimal area }-R 1 \text { intimal area }) / R 1 \text { intimal area } .
$$

\section{Immunofluorescence Analyses}

Vessel sections were obtained and embedded in paraffin. Paraffin from 5 - $\mu \mathrm{m}$-thick sections was removed with xylene, and the sections were rehydrated through graded alcohol to water. Before labeling, the sections were permeabilized for 10 minutes in phosphate-buffered saline (PBS) containing $0.1 \%$ Triton. The sections were then blocked with PBS containing 5\% bovine serum albumin for 1 hour. The sections were incubated for 1 hour at room temperature with mouse anti- $\alpha$-smooth muscle actin antibody diluted 1:100, mouse anti-desmin antibody diluted 1:200, and mouse anti-vimentin antibody diluted 1:100, which are specific for SMCs. ${ }^{12}$ After washing, the sections were incubated for 1 hour at room temperature with an anti-mouse (donkey) antibody conjugated with $\mathrm{Cy} 3$ diluted 1:400. After washing, the sections were mounted and examined under a Zeiss Axiovert microscope (Zeiss, Le Pecq, France). Control experiments with secondary antibody without primary antibody were also performed to determine nonspecific binding. 


\section{SMC Cultures}

SMCs were cultured from explants, as previously described. ${ }^{13}$ In brief, vessels were kept in DMEM at $4{ }^{\circ} \mathrm{C}$ before their intimal cell layer and residual adventitial tissue were stripped off. The dissected media of the vessels was then cut into small pieces (3-5 mm), which were transferred into cell-culture flasks. The vessel tissues were incubated in DMEM supplemented with $10 \%$ FCS, 2 mmol/L L-glutamine, and antibiotics $(100 \mathrm{U} / \mathrm{mL}$ penicillin and $0.1 \mathrm{mg} / \mathrm{mL}$ streptomycin) to allow the SMCs to grow out. After 2 weeks of incubation at $37^{\circ} \mathrm{C}$ and under a $5 \%$
$\mathrm{CO}_{2}$ humidified atmosphere, the SMCs collected in the culture medium, and the vessel tissues were transferred into new cellculture flasks. Cultured SMCs were used for experiments between passages 1 to 3 .

\section{Measurement of SMC Proliferation}

SMCs in DMEM supplemented with $10 \%$ FCS were seeded in 24-well plates at a density of $5 \times 10^{4}$ cells per well and allowed to adhere. The cells were then subjected to 48 hours of growth arrest in medium containing only $0.2 \%$ FCS and incubated in
Day 0
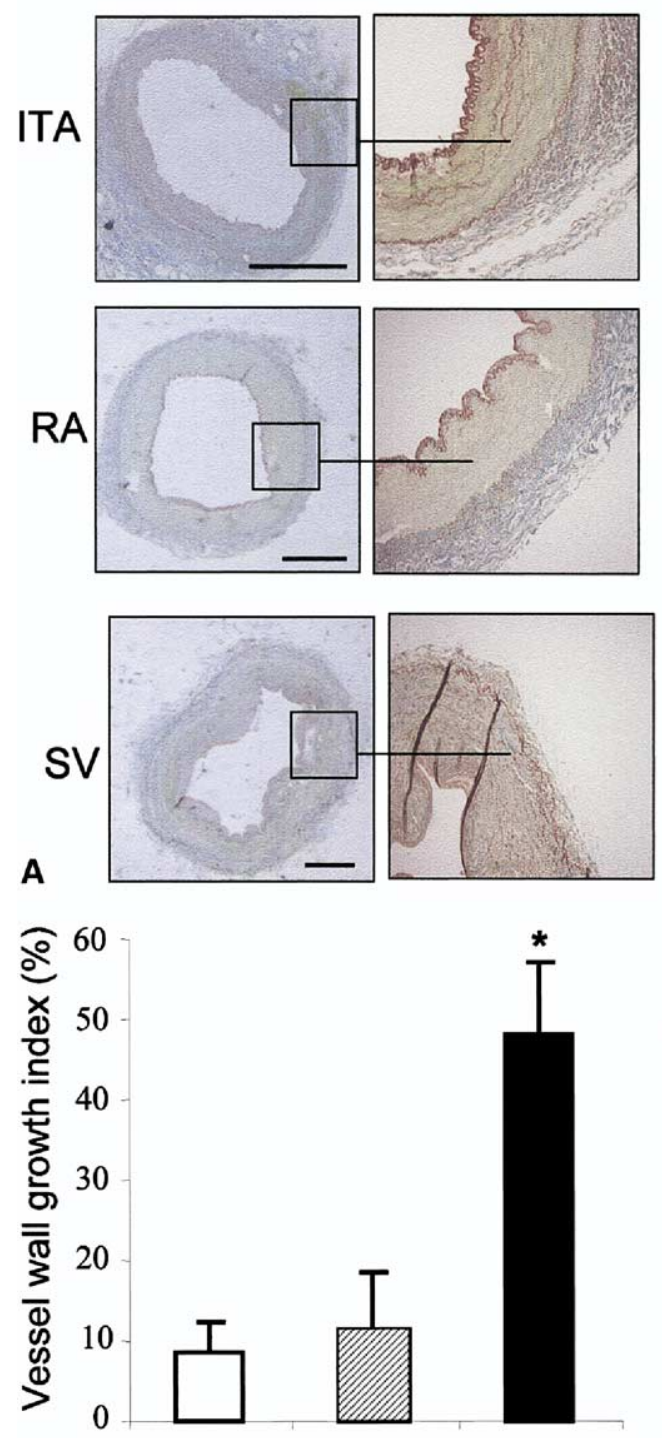

B

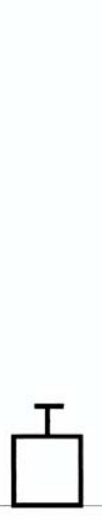

ITA

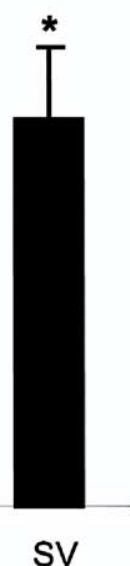

Day 10
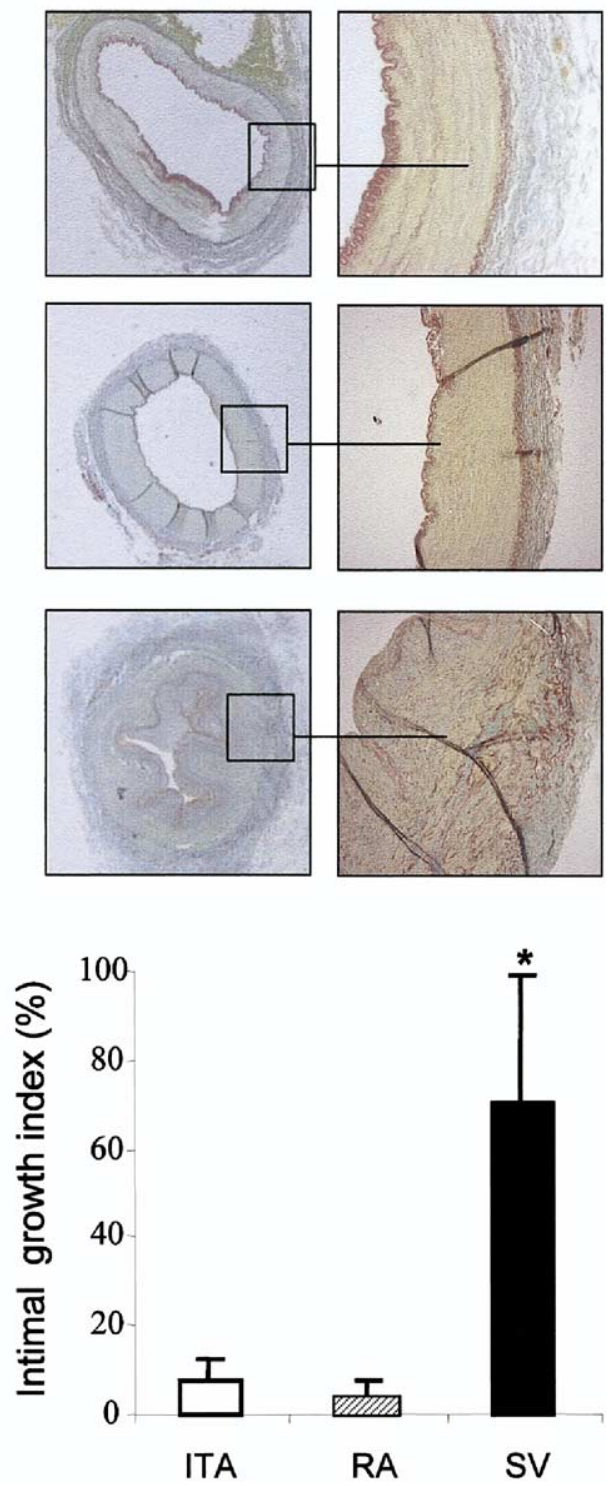

Figure 1. A, Structure and wall thickness of internal thoracic artery (ITA), radial artery (RA), and saphenous vein (SV) segments at baseline (day 0 ) and after 10 days of incubation in culture medium (day 10). The photograph was taken after orcein-picro-indigo-carmine staining (bar $=100 \mu \mathrm{m})$. B, Vessel-wall growth index and intimal growth index of each vessel after 10 days of culture. ${ }^{*} P<.05$ compared with values obtained with ITA and RA segments $(n=7)$. 
DMEM supplemented with $0.6 \mu \mathrm{Ci} / \mathrm{mL}$ tritiated thymidine with $0.5 \%, 5 \%$, or $10 \%$ FCS. After incubation for 24 hours, the cells were washed twice with PBS, treated with ice-cold $10 \%$ trichloroacetic acid (TCA), dissolved in $1 \mathrm{~N} \mathrm{NaOH}(0.5 \mathrm{~mL} /$ well), and neutralized with $75 \mu \mathrm{L}$ of $12 \mathrm{~N} \mathrm{HCl}$ per well. The incorporated radioactivity was then counted with a liquid scintillation counter. All experiments were run in 6 replicates.

\section{SMC Migration}

SMC migration was evaluated with a modified Boyden chamber. A polycarbonate filter with an 8.0- $\mu \mathrm{m}$ pore diameter (Costar, Cambridge, United Kingdom) coated with $5 \mathrm{mg} / \mathrm{L}$ gelatin was used to divide the upper and lower well chambers. Cultured SMCs were trypsinized and suspended in a concentration of $5 \times 10^{4}$ cells $/ \mathrm{mL}$ in culture medium containing $10 \%$ FCS. A volume of $0.2 \mathrm{~mL}$ of suspended cells was placed in the upper chamber, and $0.5 \mathrm{~mL}$ of the same culture medium was loaded in the lower chamber. After 24 hours of incubation $\left(37^{\circ} \mathrm{C}\right.$ at $5 \% \mathrm{CO}_{2}$ in air) to allow cell adhesion, the upper chamber medium was replaced with serumfree culture medium, and the lower chamber medium was replaced with culture medium containing FCS $(0.5 \%$ or $5 \%)$. After 24 hours of incubation, cells that had migrated to the bottom side of the filter were fixed with ethanol, stained with Diff Quick staining, and counted from 9 randomly chosen high-power $(125 \times)$ fields. All experiments were run in duplicate.

\section{Measurement of SMC Collagen Synthesis}

Collagen synthesis was assessed on the basis of measurement of cellular tritiated proline uptake, as previously described. ${ }^{14}$ Briefly, SMCs in DMEM supplemented with $10 \%$ FCS were seeded in 24-well plates at a density of $2 \times 10^{4}$ cells per well and allowed to adhere. The cells were then subjected to 48 hours of growth arrest in medium containing only $0.2 \%$ FCS and were then incubated in DMEM supplemented with $1 \mu \mathrm{Ci} / \mathrm{mL}$ tritiated proline (L-[4-3H(N)]-proline) with $0.5 \%, 5 \%$, or $10 \%$ FCS. After incubation for 48 hours, the medium was removed, and ice-cold $10 \%$ TCA was added for 30 minutes at $4^{\circ} \mathrm{C}$. After 2 rinses with cold $10 \%$ TCA, the acid-precipitable material was solubilized for 2 hours in $0.5 \mathrm{~mL}$ of $0.3 \mathrm{~N} \mathrm{NaOH}$ with $0.1 \%$ sodium dodecylsulfate at $37^{\circ} \mathrm{C}$. Incorporated radioactivity (in counts per minute) in the remaining cell lysate was measured with a liquid scintillation counter. All experiments were run in 4 replicates.

\section{Chemicals}

Tritiated thymidine was from Amersham Pharmacia Biotech (Buckingham, United Kingdom). Tritiated proline was from NEN, PerkinElmer Life Sciences (Courtaboeuf, France). The primary and secondary antibodies used for immunofluorescence analyses were purchased from Dako (DakoCytomation S.A.S., Trappes, France) and Jackson Immunoresearch Inc (Cambridgeshire, United Kingdom), respectively. Antibiotics were from Laboratoires Diamant (Paris, France) and Pharmacia (Saint-Quentin-en-Yvelines, France). FCS, DMEM, and PBS were from Invitrogen (Cergy Pontoise, France). We used the same lot of FCS for the entire experiment. Formaldehyde, xylene, triton, hematoxylin and eosin, elastic stain, orcein-picroindigo-carmine, and gelatin were from Sigma-Aldrich (Sigma-Aldrich Chimie, Lyon, France).

\section{Statistical Analysis}

Statistical analysis was performed with SPSS Base 11.5 statistical software (SPSS Inc, Chicago, Ill). All data are reported as means \pm standard error of the mean. Analysis of variance was used for between-group comparisons. When analysis of variance showed a significant difference, the groups were compared with a MannWhitney nonparametric test.

\section{Results}

\section{Histopathology and Morphometric Analysis}

Figure 1 shows the organ culture results. The total vesselwall growth index was significantly higher for $\mathrm{SV}$ rings than for RA and ITA rings $(47.9 \% \pm 9.3 \%$ vs $11.6 \% \pm 7.1 \%$ and $8.4 \% \pm 3.9 \%$, respectively; $P<.05$ for both comparisons). The intimal growth index was significantly higher for SV rings than for RA and ITA rings $(70.6 \% \pm 28.1 \%$ vs $4.3 \% \pm$ $3.0 \%$ and $7.6 \% \pm 5.0 \%$, respectively; $P<.05$ for both comparisons). There was no significant difference between ITA and RA rings concerning both wall growth index values. Immunofluorescence analyses with $\alpha$-smooth muscle actin, desmin, and vimentin showed predominant involvement of SMCs in neointimal growth induced by organ culture of SV rings (Figure 2).

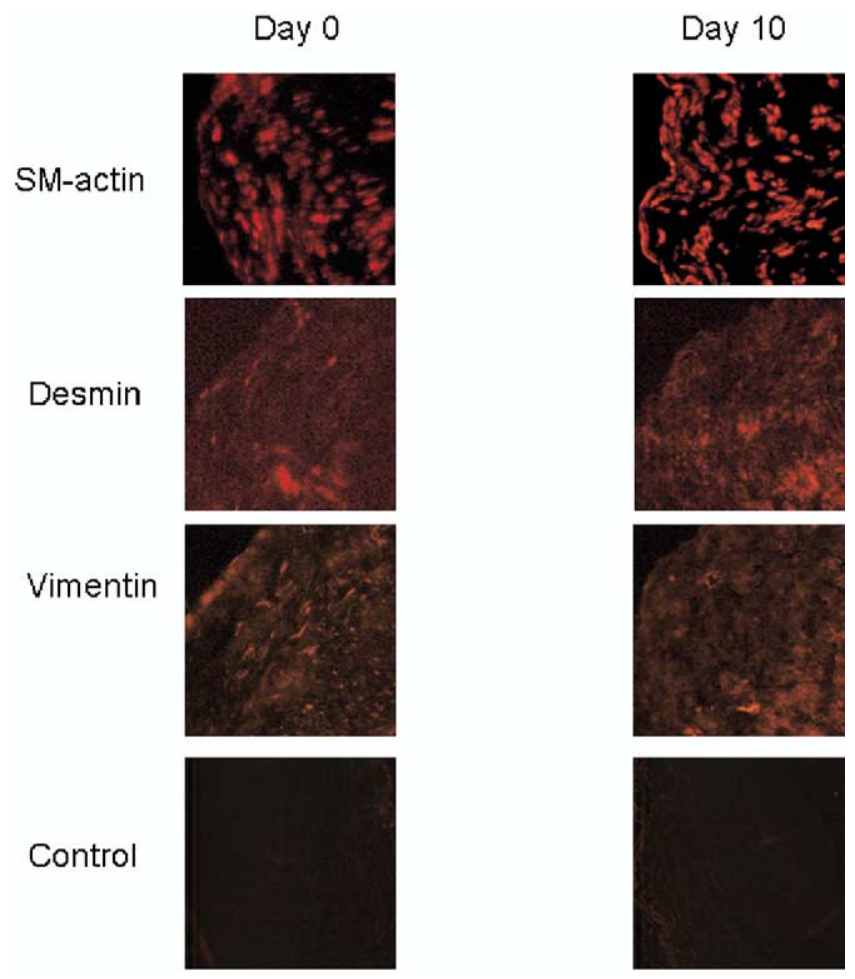

Figure 2. $\alpha$-Smooth muscle (SM) actin, desmin, and vimentin immunostaining in SV rings at baseline (day $\mathbf{0}$ ) and after 10 days of culturing. Predominant $\alpha$-smooth muscle actin immunoreactivity was noted both at baseline (day 0 ) and after 10 days of neointimal growth. 


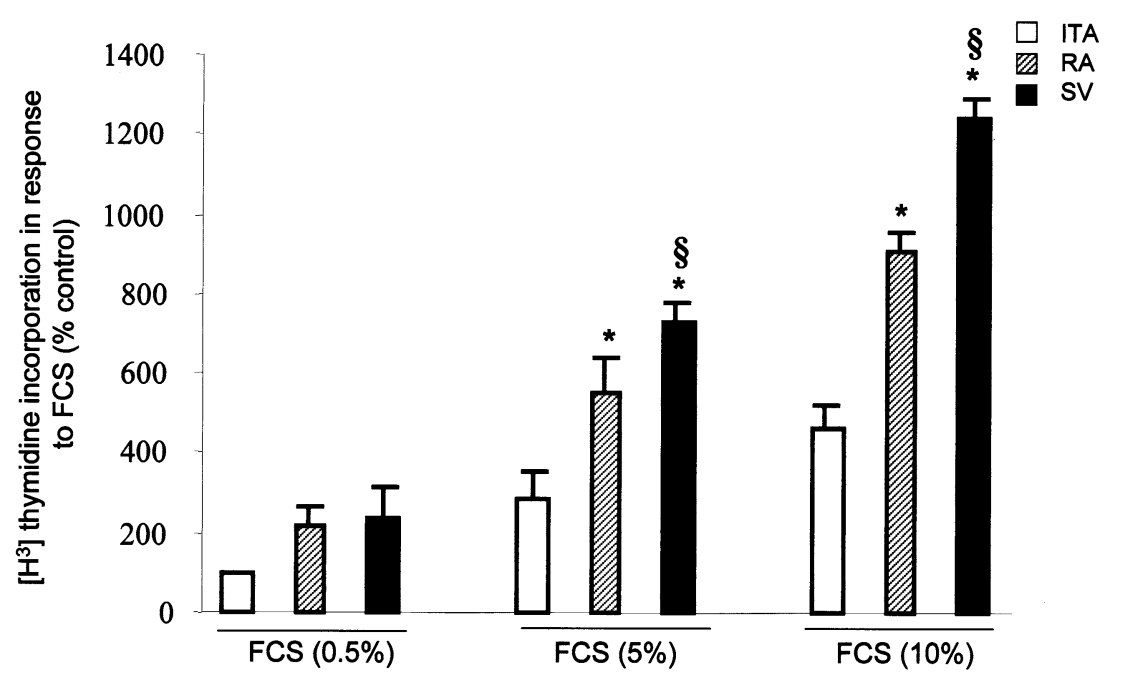

Figure 3. Stimulatory effect of increasing concentrations of fetal calf serum (FCS) on tritiated $\left(\left[{ }^{3} H\right]\right)$ thymidine incorporation in cultured smooth muscle cells from internal thoracic artery (ITA), radial artery $(R A)$, and saphenous vein (SV) rings. Values are presented as means \pm standard error of the mean. ${ }^{*} P<$ .05 versus values obtained with ITA smooth muscle cells under the same stimulation conditions. $\S P<.05$ versus values for $R A$ smooth muscle cells under the same stimulation conditions. All experiments were run in 6 replicates $(n=5)$.

\section{SMC Proliferation}

FCS stimulated proliferation of cultured SMCs from ITAs, RAs, and SVs, as demonstrated by thymidine incorporation, and the effect was greatest with 10\% FCS (Figure 3). Tritiated thymidine incorporation was significantly higher in SV SMCs than in RA and ITA SMCs independent of FCS concentration $(P<.05$ for both comparisons). In addition, tritiated thymidine incorporation in RA SMCs was significantly higher than in ITA SMCs with both $5 \%$ and $10 \%$ FCS $(P<.05$ for both comparisons $)$.

\section{SMC Migration}

SMC migration was enhanced by FCS (Figure 4). Migration of SMCs from SVs was significantly greater than that from ITAs or RAs ( $P<.05$ for both comparisons), whereas there was no significant difference between SMCs from ITAs and RAs.

\section{SMC Collagen Synthesis}

FCS stimulated tritiated $\mathrm{OH}$-proline incorporation in cultured SMCs from ITAs, RAs, and SVs, and the effect was greatest with $10 \%$ FCS (Figure 5). At each level of FCS stimulation, tritiated $\mathrm{OH}$-proline incorporation was similar in SMCs from ITAs, RAs, and SVs.

\section{Discussion}

The present study evaluated vascular remodeling in human ITA, RA, and SV segments. Cultures showed significantly greater NIH in SV rings than in ITA or RA rings. Cultured SMCs from SV grafts showed significantly greater proliferation and migration than did arterial SMCs, whereas no difference was evidenced in collagen synthesis between groups. Furthermore, SMCs from RAs exhibited significantly more proliferation than SMCs from ITAs.

$\mathrm{NIH}$ is a disease process that occurs early after grafting and affects both venous and arterial grafts. ${ }^{4}$ Although it can be viewed as adaptive, it might also predispose the graft to later accelerated graft atherosclerosis. ${ }^{15}$ In the present study, culturing vessel rings with FCS induced NIH development in all 3 types of vessels used for CABG. However, NIH was significantly less pronounced in ITA and RA rings than in $\mathrm{SV}$ rings. These results are in accordance with previous studies of human bypass graft cultures showing greater NIH severity in SV rings than in ITA rings ${ }^{16,17}$ and RA rings. ${ }^{17}$ Cable and colleagues ${ }^{17}$ have shown that organ culture of ITA and RA vascular rings for up to 14 days was not associated with significant NIH. Vascular structure is known to influence NIH development: elastic arteries are less prone to NIH than are muscular arteries. ${ }^{18}$ The ITA has a mainly elastic media with few SMCs and a dense internal elastic lamina with few fenestrations. This might be an important barrier to SMC migration and might therefore reduce the development of $\mathrm{NIH}^{1}$ In contrast, the highly fenestrated structure of the internal elastic lamina of SV grafts and the thick and muscular media of RA grafts might promote the development of NIH. ${ }^{18}$ However, there was no significant difference in NIH development between RA and ITA rings in our study.

It is generally accepted that NIH involves proliferation of SMCs in the media of the graft. These proliferating cells subsequently migrate into the graft intima, where they undergo further division and secrete extracellular matrix. ${ }^{19}$ Our organ culture model is in accordance with this pathogenic concept, as demonstrated by the major presence of SMCs with immunofluorescence analyses. SMC proliferation and migration are complex processes regulated by multiple stimulatory and inhibitory influences. Differences in endothelial function, inherent properties of SMCs, or both, might explain our findings. However, although organ culture of vascular rings maintains the anatomic relationships among cells of the vascular wall, several other variables that par- 


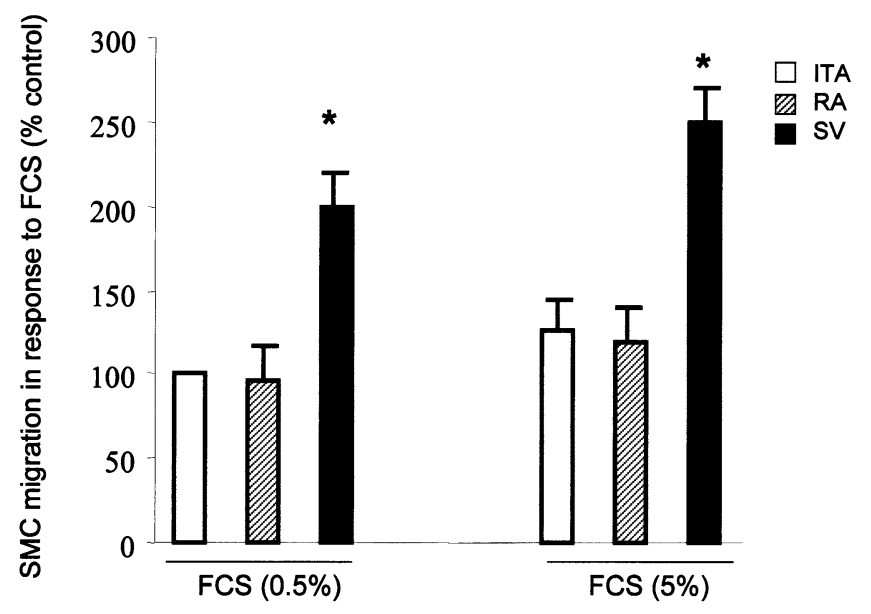

Figure 4. Smooth muscle cell (SMC) migration in response to fetal calf serum (FCS). ${ }^{*} P<.05$ versus values obtained with internal thoracic artery (ITA) SMCs and radial artery (RA) SMCs under the same stimulation conditions. All experiments were run in duplicate ( $n=4)$. $S V$, Saphenous vein.

ticipate in vivo are known to contribute to NIH (including circulating cells, hemodynamic factors, flow, and size mismatch between the conduit and the recipient artery) and are not taken into account by this model. In addition, the response to FCS might not represent the response seen in vivo. Therefore our findings might not be extrapolated to the clinical setting.

The vascular endothelium has important regulatory effects on vascular-wall remodeling. ${ }^{20}$ Several endothelium-derived mediators influence vascular SMC proliferation and migration. Thus, agents that increase SMC cyclic adenosine monophosphate concentrations (prostaglandins $E_{1}$ and $I_{2}$ ) or cyclic guanosine monophosphate concentrations (nitric oxide) have been shown to inhibit vascular SMC proliferation. ${ }^{21,22}$ ITAs release more prostaglandin $\mathrm{I}_{2}$ and show greater nitric oxide-mediated endothelium-dependent relaxation than SVs. ${ }^{1}$ Furthermore, He and $\mathrm{Liu}^{23}$ recently showed that basal and stimulated levels of nitric oxide release in human ITAs were significantly greater than those seen in RAs. This lower capacity for nitric oxide release might contribute to the susceptibility of RAs to perioperative vasospasm and might also influence the long-term patency of RA grafts by greater SMC proliferation and migration.

Alternatively, the differences in NIH across vessels could be related to differences in the inherent properties of their SMCs. Indeed, the phenotypic heterogeneity of vascular SMCs is now well established, ${ }^{11}$ and differences in proliferation and migratory properties might translate into dissimilar propensities of blood vessels to exhibit NIH. The results of our cell-culture experiments suggest that SMCs from ITAs and RAs might be more resistant to mitogenic stimulation by FCS than those from SVs, suggesting a greater propensity of SVs to enter a proliferative state. Yang and coworkers ${ }^{24}$ have shown that SMCs from SVs exhibit a higher rate of serum-induced explant outgrowth and tritiated thymidine incorporation compared with ITA SMCs. White and associates ${ }^{25}$ also evidenced a stronger tritiated thymidine incorporation response to platelet-derived growth factor (PDGF) in human SV-derived cells compared with that seen in ITA-derived cells. However, only limited data are available on the proliferative capacity of SMCs from RAs compared with those from ITAs, SVs, or both. Del Rizzo and coworkers ${ }^{26}$ reported significantly less tritiated thymidine uptake after stimulation with the PDGF-BB homodimer in SMCs from ITAs and RAs compared with those from SVs. The same investigators found higher levels of c-fos expression (an oncoprotein regulating cell proliferation and differentiation) both constitutively and after PDGF-BB stimulation in SMCs from SVs. These data suggest that SV SMCs might have a greater propensity to enter into a proliferative state compared with SMCs from RAs or ITAs. In contrast to Del Rizzo and coworkers,${ }^{26}$ we found that the proliferative response to FCS was significantly stronger in RA SMCs than in ITA SMCs. However, as noted above, this did not translate into increased NIH generation in RA rings compared with that seen in ITA rings. Considering the limited NIH production in arterial vascular grafts, ${ }^{17}$ longer incubation periods ( $>14$ days) might be necessary to show significant differences between RA and ITA vascular rings. The increased inherent propensity of RA SMCs to proliferate might explain, in addition to the previously mentioned lower nitric oxide release, the higher incidence of $\mathrm{NIH}$ at harvesting ${ }^{27,28}$ and the consistently lower long-term patency rates of RA grafts compared with left-sided ITA grafts in vivo. ${ }^{7}$

In addition to a diminished proliferative response of arterial SMCs exposed to mitogens, we found significant reductions in arterial SMC migration compared with that seen in SV SMCs. Matrix metalloproteases (MMPs) are required for NIH development, mainly because of their effects on SMC migration. Anstadt and associates ${ }^{29}$ recently reported significantly reduced MMP-2 levels and activity in ITA grafts compared with that seen in SV grafts. Whether MMP expression in RA grafts is similar to that in ITA grafts remains to be determined. However, we found no differences in migratory properties between RA SMCs and ITA SMCs in the present study.

We observed no significant differences in collagen synthesis between SV-, ITA-, and RA-derived SMCs. This observation lies in contrast with the recent report by Wong and colleagues, ${ }^{30}$ who found that venous SMCs are more dedifferentiated and demonstrate increased proliferation, migration, and synthetic capacity compared with arterial 


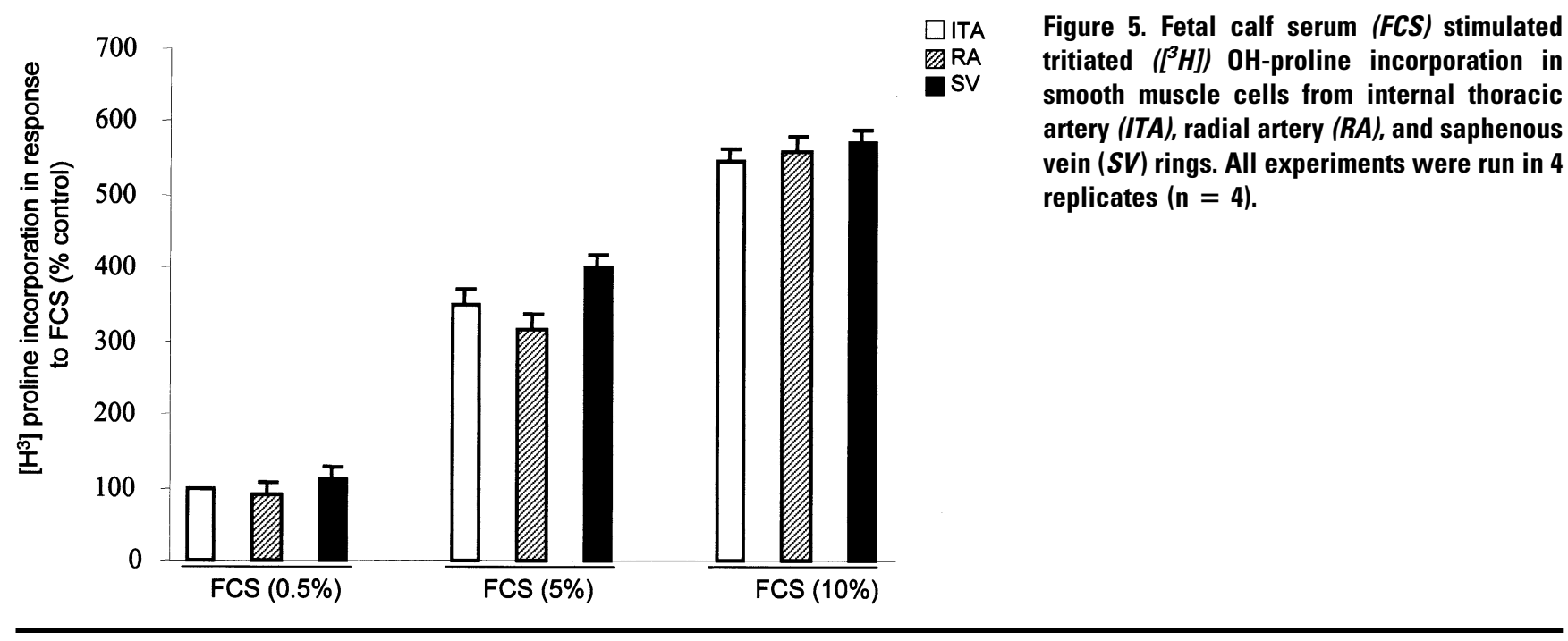

SMCs in serum-stimulated culture conditions. However, these authors used rabbit SMCs originating from carotid and jugular veins, and SMC behavior might vary in different vascular beds and species.

In conclusion, the human SV has a greater propensity for NIH formation than arterial grafts, with increased intrinsic proliferative and migratory properties of venous SMCs. In contrast, we found no significant differences in NIH formation between ITA and RA grafts, despite significantly increased proliferative properties of RAderived SMCs.

\section{References}

1. Cooper GJ, Underwood MJ, Deverall PB. Arterial and venous conduits for coronary artery bypass. A current review. Eur J Cardiothorac Surg. 1996;10:129-40.

2. Zalewski A, Shi Y, Johnson AG. Diverse origin of intimal cells: smooth muscle cells, myofibroblasts, fibroblasts, and beyond? Circ Res. 2002;91:652-5.

3. Cameron A, Davis KB, Green G, Schaff HV. Coronary bypass surgery with internal-thoracic-artery grafts-effects on survival over a 15-year period. N Engl J Med. 1996;334:216-9.

4. Curtis JJ, Stoney WS, Alford WC Jr, Burrus GR, Thomas CS Jr. Intimal hyperplasia. A cause of radial artery aortocoronary bypass graft failure. Ann Thorac Surg. 1975;20:628-35.

5. Fisk RL, Brooks CH, Callaghan JC, Dvorkin J. Experience with the radial artery graft for coronary artery bypass. Ann Thorac Surg. 1976; 21:513-8.

6. Mussa S, Choudhary BP, Taggart DP. Radial artery conduits for coronary artery bypass grafting: current perspective. J Thorac Cardiovasc Surg. 2005;129:250-3.

7. Verma S, Szmitko PE, Weisel RD, Bonneau D, Latter D, Errett L, et al. Should radial arteries be used routinely for coronary artery bypass grafting? Circulation. 2004;110:e40-6.

8. Desai ND, Cohen EA, Naylor CD, Fremes SE. A randomized comparison of radial-artery and saphenous-vein coronary bypass grafts. N Engl J Med. 2004;351:2302-9.

9. Zacharias A, Habib RH, Schwann TA, Riordan CJ, Durham SJ, Shah A. Improved survival with radial artery versus vein conduits in coronary bypass surgery with left internal thoracic artery to left anterior descending artery grafting. Circulation. 2004;109:1489-96.
10. Possati G, Gaudino M, Prati F, Alessandrini F, Trani C, Glieca F, et al. Long-term results of the radial artery used for myocardial revascularization. Circulation. 2003;108:1350-4.

11. Hao H, Gabbiani G, Bochaton-Piallat ML. Arterial smooth muscle cell heterogeneity: implications for atherosclerosis and restenosis development. Arterioscler Thromb Vasc Biol. 2003;23:1510-20.

12. Owens GK. Regulation of differentiation of vascular smooth muscle cells. Physiol Rev. 1995;75:487-517.

13. Eddahibi S, Humbert M, Fadel E, Raffestin B, Darmon M, Capron F, et al. Serotonin transporter overexpression is responsible for pulmonary artery smooth muscle hyperplasia in primary pulmonary hypertension. J Clin Invest. 2001;108:1141-50.

14. Hafizi S, Wharton J, Morgan K, Allen SP, Chester AH, Catravas JD, et al. Expression of functional angiotensin-converting enzyme and AT1 receptors in cultured human cardiac fibroblasts. Circulation. 1998;98:2553-9.

15. Murphy GJ, Angelini GD. Insights into the pathogenesis of vein graft disease: lessons from intravascular ultrasound. Cardiovasc Ultrasound. 2004;2:8.

16. Huang B, Dreyer T, Heidt M, Yu JC, Philipp M, Hehrlein FW, et al. Insulin and local growth factor PDGF induce intimal hyperplasia in bypass graft culture models of saphenous vein and internal mammary artery. Eur J Cardiothorac Surg. 2002;21:1002-8.

17. Cable DG, Caccitolo JA, Caplice N, O'Brien T, Simari RD, Daly RC, et al. The role of gene therapy for intimal hyperplasia of bypass grafts. Circulation. 1999;100(suppl II):II392-6.

18. van Son JA, Smedts F, Vincent JG, van Lier HJ, Kubat K. Comparative anatomic studies of various arterial conduits for myocardial revascularization. J Thorac Cardiovasc Surg. 1990;99:703-7.

19. Motwani JG, Topol EJ. Aortocoronary saphenous vein graft disease: pathogenesis, predisposition, and prevention. Circulation. 1998;97: 916-31.

20. Barner HB. Remodeling of arterial conduits in coronary grafting. Ann Thorac Surg. 2002;73:1341-5.

21. Newby AC, Zaltsman AB. Molecular mechanisms in intimal hyperplasia. J Pathol. 2000;190:300-9.

22. Jeremy JY, Rowe D, Emsley AM, Newby AC. Nitric oxide and the proliferation of vascular smooth muscle cells. Cardiovasc Res. 1999; 43:580-94.

23. He GW, Liu ZG. Comparison of nitric oxide release and endotheliumderived hyperpolarizing factor-mediated hyperpolarization between human radial and internal mammary arteries. Circulation. 2001; 104(suppl I):I344-9.

24. Yang Z, Oemar BS, Carrel T, Kipfer B, Julmy F, Luscher TF. Different proliferative properties of smooth muscle cells of human arterial and venous bypass vessels: role of PDGF receptors, mitogen-activated 
protein kinase, and cyclin-dependent kinase inhibitors. Circulation. 1998;97:181-7.

25. White PJ, Kumari R, Porter KE, London NJ, Ng LL, Boarder MR. Antiproliferative effect of UTP on human arterial and venous smooth muscle cells. Am J Physiol Heart Circ Physiol. 2000;279:H2735-42.

26. Del Rizzo DF, Yurkova N, Moon MC, Litchie B, Zahradka P. Plateletderived growth factor-induced expression of $\mathrm{c}$-fos in human vascular smooth muscle cells: implications for long-term graft patency. Ann Thorac Surg. 2002;74:90-5.

27. Kaufer E, Factor SM, Frame R, Brodman RF. Pathology of the radial and internal thoracic arteries used as coronary artery bypass grafts. Ann Thorac Surg. 1997;63:1118-22.
28. Ruengsakulrach P, Sinclair R, Komeda M, Raman J, Gordon I, Buxton B. Comparative histopathology of radial artery versus internal thoracic artery and risk factors for development of intimal hyperplasia and atherosclerosis. Circulation. 1999;100(suppl II): III39-44.

29. Anstadt MP, Franga DL, Portik-Dobos V, Pennathur A, Bannan M, Mawulawde K, et al. Native matrix metalloproteinase characteristics may influence early stenosis of venous versus arterial coronary artery bypass grafting conduits. Chest. 2004;125:1853-8.

30. Wong AP, Nili N, Strauss BH. In vitro differences between venous and arterial-derived smooth muscle cells: potential modulatory role of decorin. Cardiovasc Res. 2005;65:702-10. 